\title{
Diffusion multiple de la lumière : application à l'orientation de fibres dans des matériaux composites
}

\author{
J.N. Le Toulouzan et D. Wysoczanski* \\ Laboratoire d'Énergétique des Systèmes et Procédés, INSA de Rouen, URA 230 du CNRS, \\ CORIA, B.P. 08, 76131 Mont-Saint-Aignan, France \\ * Institute of Electrical Metrology, Technical University of Wroclaw, ul. B. Prusa 53/55, \\ 50-317 Wroclaw, Poland
}

\begin{abstract}
Résumé : Un diagnostic optique simple, basé sur la diffusion de la lumière, permet de mesurer de façon indépendante la concentration et l'orientation de fibres dans un matériau polymérique composite. Un système a caméras enregisure les images de diffusion données par le matériau éclairé par un faisceau laser. Le traitement des images qui ont une forme elliptique conduit aux deux paramètres concentration et orientation des fibres. La méthode de mesure est validée avec des échantillons tests constitués par une matrice polymérique contenant des fibres de verre.
\end{abstract}

\section{INTRODUCTION}

Dans de nombreuses situations industrielles ou même académiques, les milieux diphasiques ne comportent pas toujours des particules sphériques. Il est souvent intéressant de connaître la distribution d'orientation et la concentration des particules en particulier dans le cas de particules allongées qui peuvent être modélisées par des cylindres. Différentes techniques basées sur différents phénomènes physiques existent telles que la thermographie laser [ 1,2 ] les micro-ondes [ 3 ] et la diffusion de la lumière [ 4 ]. Dans le domaine des matériaux plastiques renforcés par des fibres, les techniques suivantes sont appliquées : photomicrographie [ 5,6$]$, turbidimétrie spectrale [ 7 ] et polarisation de la lumière diffusée [ 8 ].

\section{MONTAGE EXPÉRIMENTAL}

Un système de deux caméras mesure l'intensité diffusée par l'échantillon pour deux états de polarisation croisés de la lumière. Un faisceau laser $(\lambda=514 \mathrm{~nm})$, polarisé linéairement suivant la verticale et épuré par un filtre spatial, est focalisé sur l'échantillon avec une tache de diamètre $8 \mu \mathrm{m}$ sur une distance de Rayleigh de $0.1 \mathrm{~mm}$. Un système imageur, comportant un prisme de Glan pour séparer les deux états de polarisation de la lumière, forme les deux images polarisées sur deux caméras $C C D(8,8 \mathrm{~mm} * 6.6 \mathrm{~mm})$ reliées à une station IPX SPARC par l'intermédiaire d'une carte d'acquisition S2200. Les images enregistrées présentent une forme elliptique et le programme fittec optimise la « meilleure » ellipse pour une gamme donnée de niveaux de gris compris entre les niveaux 0 et 255 . A partir de cette ellipse, l'orientation du grand axe de l'ellipse détermine l'orientation des fibres, perpendiculaires à cet 
axe et la concentration en fibres est proportionnelle à la longueur du grand axe de l'ellipse [9]. Cette deuxième mesure nécessite un étalonnage avec un échantillon pour obtenir une mesure absolue en concentration.

\section{RÉSULTATS EXPÉRIMENTAUX}

La méthode est appliquée dans le cas d'un échantillon multicouche comportant des fibres longues de verre incluses dans une matrice de résine epoxy (ISRIM VE10L) [ 10 ]. La structure de l'échantillon est représentée sur la figure 1 où les cotes sont en millimètres. L'échantillon comporte 9 zones. la première zone contient une couche de fibres orientées verticalement et neuf couches orientées horizontalement $(1 \mathrm{v} 9 \mathrm{~h})$. La deuxième zone contient deux couches de fibres orientées verticalement et huit couches orientées horizontalement $(2 \mathrm{v} 8 \mathrm{~h})$, etc.

La figure 2 représente la somme des images enregistrées par les deux caméras suivant les deux états de polarisation et correspondant au milieu de chacune des zones 1 à 9 . Les images elliptiques confirment la présence des fibres orientées verticalement pour la figure $2 \mathrm{a}$ ou horizontalement pour la figure $2 \mathrm{e}$. L'image est intermédiaire entre les cas précédents lorsque le nombre de couches orientées verticalement et horizontalement est le même (figure $2 \mathrm{c}$ ).

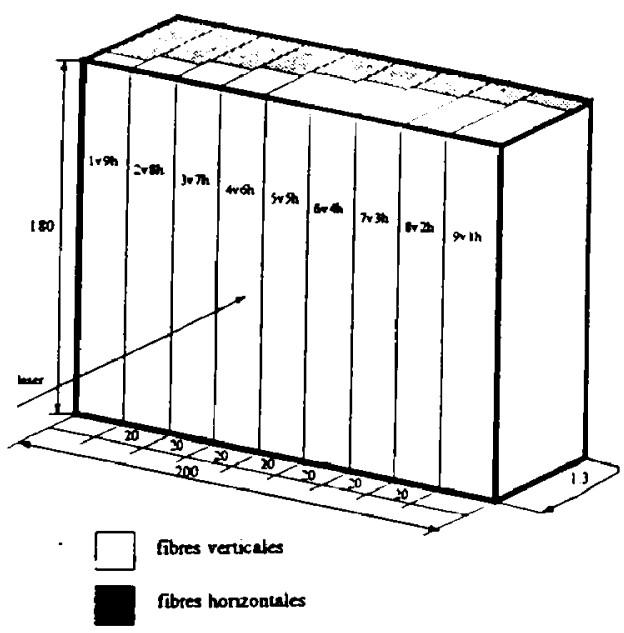

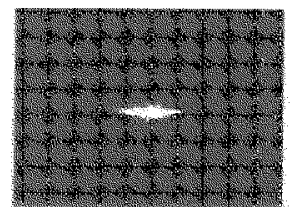

atimi d

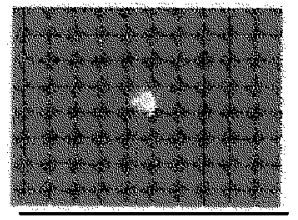

arom,

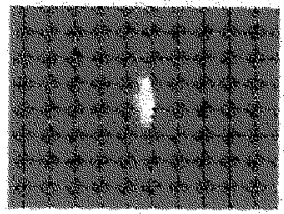

aPuns

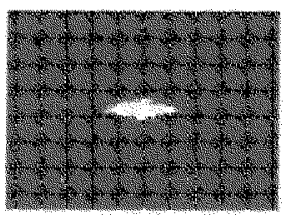

mininis

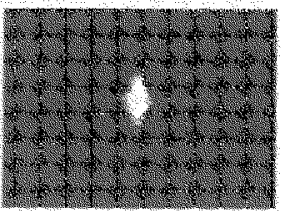

A) P., yns
Figure 1 : Echantillon ISRIM VE10L.
Figure 2 : Somme des images enregistrées par les deux caméras pour différents points de mesure de l'échantillon ISRIM L 


\section{CONCLUSION}

Ce travail montre une approche de nuages de particules fibreuses par la méthode de diffusion de la lumière. Elle est directe (sans inversion), séparée (concentration et orientation de fibres) et instantanée permettant une analyse en ligne de production. La principale limitation c'est la nécessité d'avoir des milieux « transparents » dont la transmittance est supérieure à $10^{-3}$ Nous n'avons présenté ici qu'un seul échantillon, mais notre étude a porté sur de nombreux autres échantillons variés et complexes et de différentes provenances (ISRIM, BAYER,...). Il est important de noter que la même technique s'applique à tout ensemble de fibres.

\section{Remerciements}

Ce travail s'est inscrit dans le cadre d'un contrat Brite-Euram II $n^{\circ}$ BRE2-CT92-0139 (Proposition 5081-91).

\section{Références}

[1] Busse G., Wu D. and Karpen W., Thermal-wave imaging with phase sensitive modulated thermography, J. Appl. Phys., 71 (1992), 3962-3965,.

[2] Krapez J.C., Thermal ellipsometry applied to the evalution of fibre orientation in composites, Joumée d'étude SFT sur la thermographie quatitative, Paris, 7 Déc. 1994.

[3] Bevis M.J., Allan P.S., Holden A., Busse G. and Diener L., Monitoring the multiple live feed moulding process with microwave orientation imaging, translated from Kunstoffe German Plastics, 82 (1992), 135-138.

[4] Girasole T., Gouesbet G., Gréhan G., Le Toulouzan J.N., Mroczka J., Ren K.F. and Wysoczanski D., Proceedings of the 4th International Congress On Optical Particle Sizing (PARTEC 95), Nurmberg, Germany, 21-23 Mars 1995.

[5] Mc Gee S.H. and Mc Cullough R.L., Characterization of fibre orientation in short-fibre composites, J. Appl. Phys., 55 (1984), 1394-1403

[6] O' Connell P.A.and Duckett R.A., Measurements of fibre orientation in short-fibrereinforced thermoplastics, Composites Science and Technology, 42 (1991), 329-347.

[7] Cielo P., Favis B.D. and MaldagueX., Light-scattering characterization of polyblends in the presence of multiple-scattering conditions, Polymer Engineering and Science, 27 (1987), 1601-1610,

[8] Videen G. and Bickel W. S., Light-scattering Mueller matrix for a rough fibre, Applied Optics, 31 (1992), 3488-3492,.

[9] Girasole T., Gouesbet G., Gréhan G., Le Meur F., Le Toulouzan J.N., Mroczka J., Ren K.F. and Wysoczanski D., Cylindrical fibre orientation analysis by light scattering-part2 : Experimental aspects. Summitted to Part. Part. Syst. Charac.

[10] ISRIM Scarl Instituto Superiore di Recerca e Formazione sui materiali Speciali per le technologie Avanzate. Loc. Pentima Bassa 2105100 TERNI. ITALY. 